\title{
Substrate Inhibition by the Blockage of Product Release and Its Control by Tunnel Engineering
}

Received 00th January 20xx, Accepted 00th January $20 x x$ DOI: $10.1039 / x 0 x x 00000 x$

\author{
Piia Kokkonen ${ }^{* a}$, Andy Beier ${ }^{* a, b}$, Stanislav Mazurenko ${ }^{a}$, Jiri Damborsky ${ }^{a, b}$, David Bednar ${ }^{a, b_{+}}$, Zbynek \\ Prokop $^{\mathrm{a}, \mathrm{b}_{+}}$
}

\begin{abstract}
Substrate inhibition is the most common deviation from Michaelis-Menten kinetics, occurring in approximately $25 \%$ of known enzymes. It is generally attributed to the formation of an unproductive enzyme-substrate complex after the simultaneous binding of two or more substrate molecules to the active site. Here, we show that a single point mutation (L177W) in the haloalkane dehalogenase LinB causes strong substrate inhibition. Surprisingly, a global kinetic analysis suggested that this inhibition is caused by binding of the substrate to the enzyme-product complex. Molecular dynamics simulations clarified the details of this unusual mechanism of substrate inhibition: Markov state models indicated that the substrate prevents the exit of the halide product by direct blockage and/or restricting conformational flexibility. The contributions of three residues forming the possible substrate inhibition site (W140A, F143L and I211L) to the observed inhibition were studied by mutagenesis. An unusual synergy giving rise to high catalytic efficiency and reduced substrate inhibition was observed between residues L177W and I211L, which are located in different access tunnels of the protein. These results show that substrate inhibition can be caused by substrate binding to the enzyme-product complex and can be controlled rationally by targeted amino acid substitutions in enzyme access tunnels.
\end{abstract}

\section{Introduction}

Substrate inhibition (SI) is a common phenomenon affecting about $25 \%$ of known enzymes. ${ }^{1-3}$ Instead of reaching a steadystate equilibrium at the maximum reaction speed, the excess substrate starts inhibiting the enzyme-catalyzed reaction. $\mathrm{SI}$ is often claimed to be an artefact caused by the use of artificially high substrate concentrations in enzymatic assays. However, multiple physiological phenomena are controlled by SI. Perhaps the most common example of SI comes from housekeeping metabolism, where high levels of ATP suppress glycolysis (and thus halt ATP production) by inhibiting phosphofructokinase.1,4 Other examples include the processes that maintain constant levels of neurotransmitters, rapidly terminate neural signals, maintain stable folate levels even under conditions of folate deprivation, and prevent de novo DNA methylation during cell replication. ${ }^{1}$

The most commonly invoked model of $\mathrm{SI}$ is that proposed by Haldane, in which there are two substrate-binding sites, only one of which is catalytic. ${ }^{5}$ Binding of the substrate to the

a. Loschmidt Laboratories, Department of Experimental Biology and RECETOX Faculty of Science, Masaryk University, Kamenice 5/A13, 62500 Brno, Czech Republic.

b. International Clinical Research Center, St. Anne's University Hospital Brno, Pekarska 53, 65691 Brno, Czech Republic.

* P.K. and A.B. contributed equally.

† Authors for correspondence: David Bednar, 222755@mail.muni.cz, ORCID 0000 0002-6803-0340; Zbynek Prokop, zbynek@chemi.muni.cz, ORCID 0000-0001-93584081.

Electronic Supplementary Information (ESI) available. See DOI: 10.1039/x0xx00000x allosteric binding site in the empty enzyme or the enzymesubstrate complex forms an inhibitory complex in which the catalyzed reaction is either very slow or completely stalled. Most published studies on SI have supported this allosteric mechanism ${ }^{6-17}$ or not discussed mechanistic details at all. ${ }^{18-27}$ The kinetics of Haldane's traditional mechanism in which excess substrate binds to the enzyme-substrate complex is readily described using a minimal steady-state model (Scheme 1), where an enzyme $E$ can bind one (ES) or two (SES) substrate molecules (S). The equilibrium is described by the Michaelis constant $K_{\mathrm{m}}$ and the dissociation constant for the inhibitory complex $K_{\mathrm{SI}}$.

However, it is often noted that steady-state analysis provides

$$
\begin{gathered}
\mathrm{E}+\mathrm{S} \stackrel{K_{\mathrm{m}}}{\rightleftarrows}[\mathrm{ES}) \stackrel{k_{\mathrm{cat}}}{\longrightarrow} \mathrm{E}+\mathrm{P} \\
+\mathrm{S} \\
\uparrow \downarrow k_{\mathrm{SI}} \\
\mathrm{SES}
\end{gathered}
$$

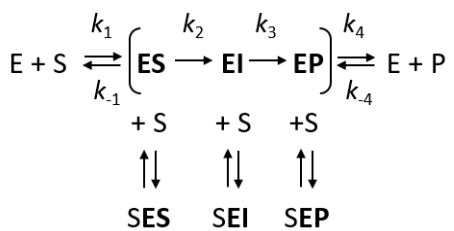

\section{Scheme 1}

\section{Scheme 2}

only indirect information and cannot be considered to provide strong support for any particular complete reaction pathway 28,29 because the steady-state parameters are complex functions of elementary steps along the kinetic pathway that are buried within these terms and cannot be individually resolved. These limitations can be avoided by examining the reaction pathway using transient-state kinetic methods that provide more detailed information on individual steps. Such 
methods can provide data on complete reaction pathways (Scheme 2), enabling investigation of other ways in which excess substrate may affect the reaction velocity. For example, excess substrate molecules may interact with enzyme forms other than the enzyme-substrate complex, such as the reaction intermediate (EI) or the enzyme-product complex (EP) to form the SEI, or SEP complexes, respectively. Transient kinetic experiments have been used in several studies to obtain detailed mechanistic insights into $\mathrm{SI}$ in multi-substrate reactions. The inhibitory effects of substrates in these cases were attributed to an accumulation of a catalytically incompetent assembly of the enzyme, cofactor, and substrate ${ }^{30}$ or, more interestingly, to the formation of a mismatched enzyme-substrate-product complex. ${ }^{31,32}$ While such mismatched formation of abortive complexes has been reported for multi-substrate and multi-product enzymes with multiple binding sites, 33,34 no comparable mechanism of substrate inhibition has yet been described for single substrate enzymes, where the formation of an enzyme-substrate-product complex is considered less likely.

Here we present a study on $\mathrm{SI}$ in the enzyme-product complex of haloalkane dehalogenase LinB from Sphingobium japonicum UT26, which sheds further light on this unusual mechanism of SI. We initially studied the wild type enzyme and two variants with engineered access pathways. Disturbing the transport of ligands into and out of the active site by blocking the main tunnel with a bulky Trp residue (L177W) unexpectedly caused strong SI. ${ }^{35,36}$ To investigate this effect, a new variant (LinBW140A/F143L/L177W/I211L) bearing three further mutations was generated. These three mutations opened an auxiliary access tunnel to the active site, modified the dynamics of the main tunnel, and restored the level of SI to that observed in the wild type. Here, we delve deeper into the mechanisms of the $\mathrm{SI}$ in these LinB variants using molecular dynamics simulations and steady-state and transient kinetics experiments. We also characterize three new LinB variants (W140A/L177W, F143L/L177W, and L177W/I211L) to determine how $\mathrm{SI}$ is affected by the individual mutations in the quadruple mutant. In addition to revealing an unusual mechanism of SI, this is the first study to combine molecular dynamics simulations with Markov state models (MSM) to investigate enzyme inhibition mechanisms.

\section{MATERIALS AND METHODS}

\section{Computational methods}

SYSTEM PREPARATION. Crystal structures (PDB IDs 1MJ5 (WT), 4WDQ (L177W) and 5LKA (W140A/F143L/L177W/I211L)) were downloaded from the RSCB Protein Data Bank. ${ }^{37-39}$ Extra ligands and salt ions were removed and hydrogen atoms were added to the structures at $\mathrm{pH} 7.5$ using the $\mathrm{H++}$ webserver. ${ }^{40}$ Water molecules in the crystal structures that did not overlap with the protonated structures were retained. The W140A/L177W, L177W/I211L, and F143L/L177W mutants were generated from the 4 WDQ structure using the PyMOL Mutagenesis Wizard, choosing the most probable side-chain orientations. ${ }^{41}$ The products, $\mathrm{BRE}$ and the bromide ion $\left(\mathrm{Br}^{-}\right)$, were manually placed in the active site and a DBE molecule was manually placed near the entrance of each enzyme's main tunnel using PyMol. ${ }^{41}$ Force field parameters and partial charges for DBE and BRE were determined using the parameterize tool of High-Throughput Molecular Dynamics (HTMD) with the default settings and the GAFF2 force field. ${ }^{42}$

The systems were solvated in a cubical water box of TIP3P water molecules so that all atoms were at least $10 \AA$ from the surface of the box using the ember build module of HTMD with the Amber FF14SB force field for the protein. ${ }^{42} \mathrm{Cl}^{-}$and $\mathrm{Na}^{+}$ions were added to neutralize the protein's charge and establish a final salt concentration of $0.1 \mathrm{M}$. The charges of all ions were scaled by a factor of 0.7 in the parameter file to counter the missing polarization effect of the force field. ${ }^{43}$ Without this scaling step, the bromide ion left the active site during the equilibration steps or at the start of the first epoch of the simulations.

SYSTEM EQUILIBRATION. The systems were equilibrated using the last step of the Equilibration_v2 protocol of HTMD. The equilibration step started with a 500-step conjugate gradient minimization. Then the system was heated and equilibrated as follows: (I) $2.5 \mathrm{~ns}$ of NPT equilibration with the Langevin thermostat at $300 \mathrm{~K}$ with $1 \mathrm{kcal} . \mathrm{mol}^{-1} . \AA^{-2}$ constraints on all heavy atoms of the protein, followed by (II) $2.5 \mathrm{~ns}$ of NPT equilibration with the Langevin thermostat at $300 \mathrm{~K}$ without constraints. ${ }^{42}$ During these equilibration simulations, holonomic constraints were applied to all hydrogen-heavy atom bond terms and the mass of hydrogen atoms was scaled by a factor of 4 , enabling the use of a $4 \mathrm{fs}$ timestep. ${ }^{44-47}$

AdaPtive SAMPling - HTMD \& ACEMD. The production simulations were run with adaptive epochs of $10 * 50 \mathrm{~ns}$ in NPT at $300 \mathrm{~K}$ using the default settings of the HTMD Production_v6 protocol. The metric used during adaptive sampling was the distance from the $N^{\varepsilon}$ of the halide-stabilizing tryptophan to the closest atoms of DBE, BRE, and the bromide ion. The total simulation times were $24,000 \mathrm{~ns}, 23,900 \mathrm{~ns}$ and $24,450 \mathrm{~ns}$ for WT, L177W, and W140A/F143L/L177W/I211L, respectively, and 25,000 ns for W140A/L177W, L177W/I211L, and F143L/L177W.

MARKOV STATE MODEL (MSM) CONSTRUCTION - HTMD. The Markov state models were built by generating a binary contact map of the three ligands (DBE, BRE, bromide) and the protein's $\mathrm{C} \alpha$ atoms with an $8 \AA$ threshold. 3-dimensional TICA was used to find correlations of states in time with a 5 ns lag time. ${ }^{48}$ The data were clustered into 200 clusters using the MiniBatchKmeans algorithm. An implied timescale plot was constructed to select a lag time for Markov model construction. The timescales stabilized when the lag time was set to 20 ns (Supplementary Figure S2), so this value was used in the models to construct the 8 Markov states. The Chapman-Kolmogorov test was used to ensure the quality of the obtained MSMs (Supplementary Table S3).

Multivariate Data ANAlysis. A partial least squares (PLS) analysis ${ }^{49}$ was performed to explore the relationships between the probabilities obtained from the molecular dynamics simulations $(\mathrm{X})$ and the experimentally determined $K_{\mathrm{i}} / K_{\mathrm{m}}$ 
constants (y) (Supplementary Figure S11 and S12). PLS reveals the correlation structure between variables $\mathrm{X}$ and $\mathrm{y}$ by reweighting the $X$ variables using PLS weights and projecting them onto a smaller number of new latent variables. Autoscaled and centered data were used in the PLS analysis. The complete data matrix used in the PLS analysis is provided in Table X. The importance of each descriptor in the model was assessed by computing the variable importance in the projection (VIP) parameter ${ }^{50}$ and plotting the PLS variable weights. ${ }^{51}$ Crossvalidation and permutation testing were performed to assess the quality and validity of the developed PLS models. ${ }^{52}$ In crossvalidation, ${ }^{50}$ some $Y$ data is excluded during model development and the resulting model is used to predict the excluded data; the predictions are then compared to the real values, and the cross-validated $\mathrm{Q}^{2}$ value is computed. This provides a more realistic assessment of predictive power than the squared multiple regression coefficient $R^{2}$. In this work, 1/7 of the compounds were deleted in each cross-validation round. In the permutation testing, the model was recalculated 300 times by randomly re-ordering the dependent variable $y$. Statistical analyses were performed with the statistical package SIMCA-P version 12 (Umetrics, Umea, Sweden) and Matlab R2019b (The MathWorks, Inc., Natick, Massachusetts, United States).

Because of the small number of available observations, our permutation analysis was reinforced by performing random Monte Carlo simulations. We generated a probability matrix and kinetic constants of the same sizes as in our analysis that were drawn randomly and independently from a uniform distribution 10,000 times. The PLS analysis was repeated for each drawing to calculate $R^{2}$ and $Q^{2}$ values, including the selection of the 3 most important descriptors (out of 12) in the models based on their VIP values and repeated the PLS.

\section{Experimental methods}

Chemicals AND GROWTH MEDIA. 1,2-dibromoethane (DBE) and LB medium were purchased from Sigma-Aldrich Co. (St. Louis, MO, USA). IPTG was purchased from Duchefa Biochemie B.V. (Haarlem, The Netherlands). All chemicals used in this study were of analytical grade.

STRAINS. Escherichia coli Dh5a cells were obtained from Invitrogen and Escherichia coli BL21 (DE3) from New England Biolabs.

Site-diReCTED MUTAGENESIS. The gene encoding LinB (from Sphingobium japonicum UT26) on the $\mathrm{pET} 21 \mathrm{~b}$ vector (Invitrogen) was used as a template to synthesize the LinB mutants. Primers were designed using the one-click web server (http://tucksengwong.staff.shef.ac.uk/OneClick/index) and then modified to be suitable for the synthesis of megaprimers (Supplementary Table S5). A standard pET vector primer was used as a reverse primer for megaprimer generation.

Site-directed mutagenesis was performed using a megaprimer mutagenesis protocol. First, a PCR to generate the megaprimers was performed. The PCR program started with $30 \mathrm{~s}$ at $98{ }^{\circ} \mathrm{C}$, followed by 30 cycles of denaturation $\left(98^{\circ} \mathrm{C}, 10 \mathrm{~s}\right)$, annealing $\left(55^{\circ} \mathrm{C}, 20 \mathrm{~s}\right)$, and extension $\left(72{ }^{\circ} \mathrm{C}, 30 \mathrm{~s}\right)$. Finally, the temperature was held at $72{ }^{\circ} \mathrm{C}$ for $120 \mathrm{~s}$. The reaction mix $(50 \mu \mathrm{L})$ contained 10x Phusion buffer $(5 \mu \mathrm{L})$, a mixture of deoxynucleoside triphosphates ( $1 \mu \mathrm{L}, 0.25 \mathrm{mM}$ each), Phusion DNA polymerase $(0.5 \mu \mathrm{L})$, plasmid DNA $(1 \mu \mathrm{L})$, and the forward and reverse primers $(2 \mu \mathrm{L}, 0.2 \mu \mathrm{M}$ each). After analysis by agarose gel electrophoresis, the megaprimers were used for the mutagenesis. The PCR program was almost identical to that for the first PCR, except that the extension and final extension steps were extended to $150 \mathrm{~s}$ and $480 \mathrm{~s}$, respectively. The reaction mix $(25 \mu \mathrm{L})$ contained 10x Phusion buffer $(2.5 \mu \mathrm{L})$, a mixture of deoxynucleoside triphosphates $(0.5 \mu \mathrm{L}, 0.25 \mathrm{mM}$ each), Phusion DNA polymerase $(0.25 \mu \mathrm{L})$, plasmid DNA $(0.5 \mu \mathrm{L})$, and the appropriate megaprimer $(1 \mu \mathrm{L})$. The reaction mixtures were then subjected to Dpnl digestion ( $2 \mu \mathrm{L} \mathrm{Dpnl}$ and $3 \mu \mathrm{L}$ CutSmart buffer/reaction mix, $37^{\circ} \mathrm{C}$ for $2 \mathrm{~h}$ then $65{ }^{\circ} \mathrm{C}$ for $20 \mathrm{~min}$ ) to remove the template DNA. Chemo-competent $E$. coli Dh5 $\alpha$ cells were then transformed with $10 \mu \mathrm{L}$ of the reaction solution for DNA strand repair and plasmid amplification. After plasmid isolation with the "InnuPREP Plasmid Mini Kit" (Analytik Jena), the success of the mutagenesis was confirmed by sequencing (performed by Eurofins MWG GmbH).

\section{CULTIVATION AND EXPRESSION IN E. COLI BL21 (DE3)}

The generated plasmids were used to transform chemocompetent E.coli BL21 (DE3) cells. Obtained colonies were used to prepare precultures by inoculating $10 \mathrm{~mL}$ LB medium (with $100 \mu \mathrm{g} / \mathrm{mL}$ ampicillin) followed by incubation at $37^{\circ} \mathrm{C}$ and 180 rpm overnight. To express each variant, 2 L LB medium supplemented with $100 \mu \mathrm{g} / \mathrm{mL}$ ampicillin was inoculated with $5 \mathrm{~mL}$ of the appropriate preculture (1/200). The flasks were incubated at $37{ }^{\circ} \mathrm{C}$ and $180 \mathrm{rpm}$ until $\mathrm{OD}_{600} 0.6-0.8$ was reached, then at $20^{\circ} \mathrm{C}$ for $30 \mathrm{~min}$. $\beta$-D-1-thiogalactopyranoside (IPTG, $0.2 \mathrm{mM}$ ) was added for induction, followed by incubation at $20^{\circ} \mathrm{C}$ at $180 \mathrm{rpm}$ overnight. The cultures were harvested by centrifugation at $4500 \times \mathrm{g}, 4^{\circ} \mathrm{C}$ for $15 \mathrm{~min}$ and the cell pellets were frozen at $-80{ }^{\circ} \mathrm{C}$ until further use.

\section{Protein PURification}

The cell pellets were suspended in $50 \mathrm{~mL}$ equilibration buffer (20 mM phosphate buffer $\mathrm{pH} 7.5$ containing $0.5 \mathrm{M} \mathrm{NaCl}$ and $10 \mathrm{mM}$ imidazole) and disrupted by sonication with a Hielscher UP200S ultrasonic processor (Hielscher, Germany) four times for four minutes each. Disrupted cells were centrifuged at $13000 \times \mathrm{g}$ and $4{ }^{\circ} \mathrm{C}$ for $1 \mathrm{~h}$ (Laborzentrifugen, Germany). The crude extract was collected, filtered, and loaded onto a Ni-NTA Superflow Cartridge (Qiagen, Germany) in equilibration buffer. Unbound and weakly bound proteins were washed out using increasing imidazole concentrations. The target enzyme was then eluted with purification buffer containing $300 \mathrm{mM}$ imidazole. The eluted protein was dialyzed three times overnight against $50 \mathrm{mM}$ phosphate buffer $(\mathrm{pH}$ 7.5). The purity of the protein was checked by SDS-polyacrylamide gel electrophoresis (SDS-PAGE). 15\% polyacrylamide gels were stained with Instant Blue (Fluka, Switzerland). Protein concentrations were determined using a NanoDrop spectrophotometer (Sigma-Aldrich, USA). The enzymes were lyophilized using a vacuum pump system for long-term storage. 
Circular dichroism (CD) SPECTROSCOPY. CD spectra were recorded at $20^{\circ} \mathrm{C}$ using a Chirascan spectropolarimeter (Applied Photophysics, United Kingdom). Data were collected from 190 to $260 \mathrm{~nm}$ at $100 \mathrm{~nm} / \mathrm{min}$ with a $1 \mathrm{~s}$ response time and $1 \mathrm{~nm}$ bandwidth using a $0.1 \mathrm{~cm}$ quartz cuvette. Each spectrum shown is an average of five individual scans and was corrected for the buffer's absorbance. Collected CD data were expressed in terms of the mean residue ellipticity ( $\Theta_{\mathrm{MRE}}$ ) using Equation 1, where $\Theta_{\text {obs }}$ is the observed ellipticity in degrees, $M_{\mathrm{w}}$ is the protein's molecular weight, $\mathrm{n}$ is the number of residues, $\mathrm{I}$ is the cell path length, $c$ is the protein concentration $(0.2 \mathrm{mg} / \mathrm{ml})$, and factor 100 originates from the conversion of the molecular weight to $\mathrm{mg} / \mathrm{dmol}$ (Supplementary Figure S10).

$$
\Theta_{M R E}=\frac{\Theta_{o b s} \cdot M_{W} \cdot 100}{n \cdot c \cdot l}
$$

Thermal DENATURATION. Thermal unfolding was followed by monitoring the ellipticity at $224 \mathrm{~nm}$ over the temperature range of 20 to $94{ }^{\circ} \mathrm{C}$, with a resolution of $0.1{ }^{\circ} \mathrm{C}$, at a heating rate of $1{ }^{\circ} \mathrm{C} / \mathrm{min}$. Recorded thermal denaturation curves were roughly normalized to represent signal changes between approximately 1 and 0 , and were fitted to sigmoidal curves using Origin 6.1 (OriginLab Corporation, USA). The melting temperatures $\left(T_{m}\right)$ were evaluated as the midpoints of the normalized thermal transitions (Supplementary Table S6).

SPECIFIC ACTIVITY MEASUREMENTS. Dehalogenation activity was assayed using the colorimetric method of Iwasaki et al. ${ }^{53}$ Halide ion release was analysed spectrophotometrically at 460 $\mathrm{nm}$ using an Eon microplate reader (BioTek, USA) after reaction with mercuric thiocyanate and ferric ammonium sulfate. The reactions were performed at $37^{\circ} \mathrm{C}$ in $25-\mathrm{ml}$ Reacti flasks sealed with Mininert valves. The reaction mixtures consisted of $10 \mathrm{ml}$ of $100 \mathrm{mM}$ glycine buffer $(\mathrm{pH} \mathrm{8.6)}$ and $10 \mu$ l of the substrate DBE. Reactions were initiated by adding enzymes to final concentrations of 0.0047 (L177W), 0.0029 (W140A/L177W), 0.0014 (F143L/L177W), 0.0073 (L177W/I211L), or 0.0005 $\mathrm{mg} / \mathrm{ml}$ (W140A/F143L/L177W/I211L). The reactions were monitored by withdrawing $1 \mathrm{ml}$ samples from the reaction mixture after $0,5,10,15,20$ and $30 \mathrm{~min}$. These samples were immediately mixed with $0.1 \mathrm{ml}$ of $35 \%$ nitric acid to stop the reaction. Dehalogenation activity was quantified as the rate of product formation over time. Each activity was measured in three independent replicates (Supplementary Table S7).

Steady-State Kinetic Measurements. The steady-state kinetics of DBE conversion by LinB variants were measured at $37{ }^{\circ} \mathrm{C}$ using a VP-ITC isothermal titration microcalorimeter (MicroCal, Piscataway, NJ, USA)..$^{54}$ The microcalorimeter's reaction vessel was filled with $1.4 \mathrm{~mL}$ of enzyme solution at a concentration of $0.001-0.12 \mathrm{mg} / \mathrm{mL}$ ( $100 \mathrm{mM}$ glycine buffer, $\mathrm{pH}$ 8.6). The substrate solution was prepared in the same buffer by adding DBE to a final concentration of 12-18 mM. The substrate concentration was verified by gas chromatography (Finnigen, USA). The enzyme was titrated at $150 \mathrm{~s}$ intervals in the reaction vessel with increasing amounts of the substrate while maintaining pseudo-first-order conditions. Each injection increased the substrate concentration, thereby increasing the rate of the catalyzed reaction (and the heat generated) until the enzyme became saturated. In total, 28 injections were performed during the titration. The reaction rates reached after each injection (in units of thermal power) were converted into enzyme turnover values using the apparent molar enthalpy $\left(\Delta \mathrm{H}_{\mathrm{app}}\right)$, as shown in Equation 2, where [P] is the molar concentration of product generated and $Q$ is the enzymegenerated thermal power.

$$
\begin{aligned}
\text { rate } & =\frac{d[P]}{d t}=\frac{1}{v \cdot \Delta \mathrm{H}_{a p p}} \cdot \frac{d Q}{d t} \\
\Delta \mathrm{H}_{\text {app }} & =\frac{1}{[S]_{\text {tot }} \cdot v} \int_{t=0}^{t=\infty} \frac{d Q(t)}{d t} d t
\end{aligned}
$$

Apparent molar enthalpy $\left(\Delta \mathrm{H}_{\mathrm{app}}\right)$ was determined using Equation 3, where $[\mathrm{S}]$ is the molar concentration of $\mathrm{DBE}$ converted by LinB in a separate experiment where the reaction was allowed to proceed to completion. The calculated enzyme turnover plotted against the actual concentration of the substrate after each injection was then fitted by nonlinear regression to kinetic models using Origin 8.0 (OriginLab, Northampton, MA, USA).

Global Data Analysis. The steady-state and transient kinetic data were fit globally using Global Kinetic Explorer (KinTek Corporation). Rate equations were numerically integrated by using the Bulirsch-Stoer algorithm with an adaptive step size to search for a set of kinetic parameters derived from the input model that minimized the $\chi 2$ value. Nonlinear regression to fit the data was performed using the Levenberg-Marquardt method. ${ }^{55}$ To account for variations in the data, enzyme and substrate concentrations were slightly adjusted by treating them as parameters during the fitting procedure with enforced boundaries at $\pm 5 \%$. Residuals were normalized by the sigma value for each data point. The standard error (S.E.) was calculated from the covariance matrix when performing nonlinear regression. The standard error estimates in the fitted parameters were propagated to obtain estimates of the error in the calculated values, the equilibrium dissociation constant of the substrate-inhibited complex $\left(K_{\mathrm{sl}}\right)$, the specificity constant $\left(k_{\mathrm{cat}} / K_{\mathrm{m}}\right)$, and the degree of substrate inhibition $\left(K_{\mathrm{sl}} / K_{\mathrm{m}}\right)$.

\section{RESULTS}

\section{Kinetic models and global data analysis}

The modification of the export pathways of the haloalkane dehalogenase LinB presented in our previous study showed that a single mutation blocking the main access tunnel (L177W) could induce strong $\mathrm{SI}$, which was subsequently removed by modifying the auxiliary tunnel (W140A/F143L/L177W/I211L) altering the dynamics of the main tunnel (Figure 1). ${ }^{35,36}$ To provide a deeper understanding of the mechanism of this inhibitory effect, we performed in a first step a systematic 
A

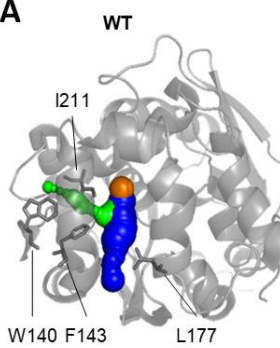

B

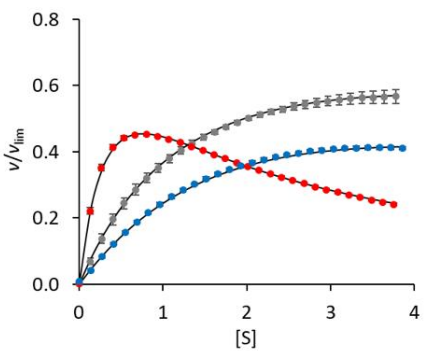

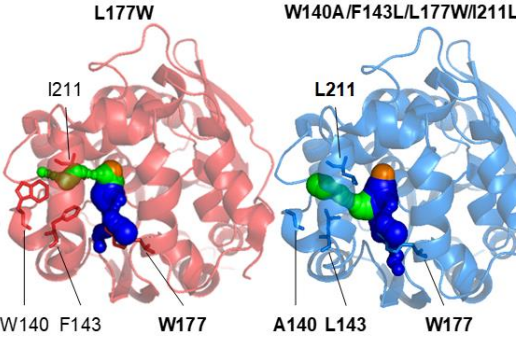

C

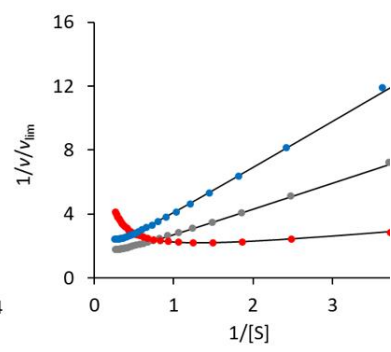

Figure 1. Overview of LinB variants with engineered access tunnels. A) The crystal structures PDB ID 1MJ5 (WT, grey), 4WDR (L177W, red) and 4WDQ (W140A/F143L/L177W/I211L, blue). The mutated residues are shown in stick form, with a bold label where mutated, and the location of the catalytic site is indicated by an orange sphere representing the co-crystallized chloride ion. The main tunnel is shown in blue. It is divided into two smaller tunnels in L177W and W140A/F143L/L177W/I211L by the introduced L177W mutation. The target of the W140A/F143L/L177W/I211L mutations, the p3 tunnel, is shown in green. B) Steady-state relative initial velocity $\left(v / v_{\text {lim }}\right)$ as a function of substrate concentration [S], and C) the Lineweaver-Burk double-reciprocal plot for the WT (grey), L177W (red) and W140A/F143L/L177W/I211L (blue) enzymes. Kinetic data were acquired in three independent replicate experiments; error bars represent the upper and lower bounds of $95 \%$ confidence intervals.

global kinetic analysis. We simultaneously fitted the steadystate and transient kinetic data by numerically integrating the rate equations derived from the minimal kinetic pathway of the HLD-catalyzed reaction (Scheme 2). Even for the simple kinetic pathway including one reaction intermediate, the excess substrate could inhibit the catalytic cycle in three ways: by interacting with the enzyme-substrate complex, the alkylenzyme intermediate, or the enzyme-product complex (Supporting Scheme S2-4). To identify the model that fits best the kinetic observations, we analyzed all three possible scenarios, examined the error in the fitted parameters and evaluated the goodness of fit visually and computationally. The normalized standard deviation $\chi 2$ and goodness of fit estimate ( $\chi^{2} /$ DoF, degrees of freedom) suggested that the model where the substrate binds to the enzyme-product complex is more likely than the other two. Detailed inspection of the fit quality showed that although the steady-state data agreed reasonably well with the simulations for all three models, there were significant discrepancies between the experimental and simulated values for both transient kinetic datasets, which were obtained by performing stopped-flow multiple turnover fluorescence and rapid-quench burst experiments. In particular, the rates and amplitudes derived from the rapid quench burst data strongly supported one mechanism for the substrate inhibition of LinB by DBE and excluded others. Specifically, the

only model consistent with the rates and amplitudes observed in all phases was that where the substrate binds to the enzymeproduct complex (Supplementary Figure S1 and Table S1). The estimates of the equilibrium dissociation constant of the substrate-inhibited complex $K_{\mathrm{SI}}$ also differed strongly between the models. The estimate for the model involving inhibitory binding of the substrate to the enzyme-product complex $\left(K_{\mathrm{SI}}=\right.$ $1.21 \pm 0.01 \mathrm{mM}$ ) agreed reasonably well with experiment, whereas the estimates for the other two models were in the sub-micromolar range. A detailed description of the global data analysis and the statistical results is provided in the Supplementary Information (Section I).

\section{Molecular dynamics simulations and Markov state models}

Because the kinetic model suggested that the observed SI was due to the formation of an enzyme-product-substrate complex (Figure 2), the starting structures for the molecular dynamics simulations consisted of systems with the two products (BRE and $\mathrm{Br}^{-}$) bound and DBE in the vicinity of the enzyme in the solvent. Also, to clarify the impact of the individual mutations differentiating L177W from W140A/F143L/L177W/I211L, the

A

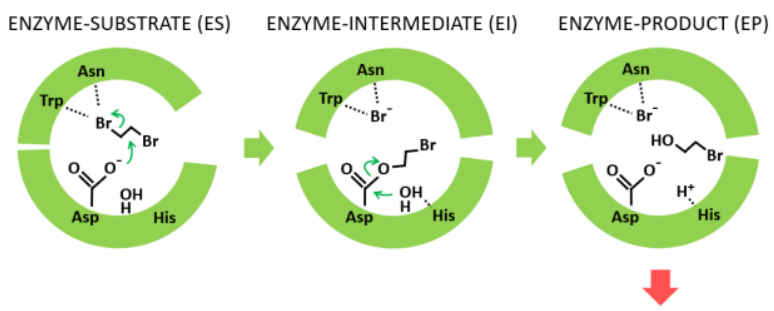

$$
\begin{aligned}
& \mathrm{B} \mathrm{E}+\mathrm{S} \stackrel{k_{\mathrm{S}}}{\rightleftarrows} \mathrm{ES} \stackrel{k_{2}}{\longrightarrow} \mathrm{El} \stackrel{k_{3}}{\rightarrow} \mathrm{EP} \stackrel{k_{4}}{\rightarrow} \mathrm{E}+\mathrm{P} \\
& + \\
& \downarrow \uparrow \kappa_{\text {S }} \\
& \underset{\text { SEP }}{\downarrow \uparrow}
\end{aligned}
$$

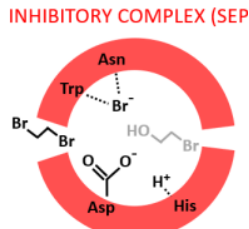

Figure 2. The mechanism of substrate inhibition. A) Scheme of the catalytic mechanism of haloalkane dehalogenases (HLDs), the active states in green and proposed SI state in red. The reaction mechanism of HLDs adapted from Verschueren et al. $1993^{56}$ is a two-step process that occurs inside the enzyme active site. A nucleophilic attack on a carbon atom is initiated by Asp, forming an ester intermediate that is then hydrolyzed by a nucleophilic attack of a water molecule activated by the basic His. Trp and Asn are halide-stabilizing residues. B) The minimal kinetic model for the catalytic cycle of HLDs (black). $S$ represents a substrate, $P$ a product, $E$ an enzyme, ES a non-covalent enzyme-substrate complex, EI a covalently bound alkyl-enzyme intermediate, and EP an enzyme-product complex. $K_{\mathrm{S}}$ is the equilibrium dissociation constant for the ES complex $\left(K_{\mathrm{S}}=k_{-1} / k_{1}\right), k_{2}$ is the rate constant for carbon-halogen bond cleavage $\left(\mathrm{S}_{N} 2\right), k_{3}$ is the rate constant for hydrolysis of the alkyl-enzyme intermediate via nucleophilic addition $\left(\operatorname{Ad}_{N}\right)$, and $k_{4}$ is the rate constant for product release. The mechanism of substrate inhibition (in red) is based on a global kinetic data analysis. $K_{\mathrm{SI}}$ is the equilibrium dissociation constant for the inhibitory enzyme-substrate-product complex (SEP)

corresponding double-point mutants were simulated (W140A/L177W, F143L/L177W and L177W/I211L). Each system was simulated using AceMD for $\sim 25 \mu$ s using the distances of the three ligands to the catalytic site as the metric for adaptive 


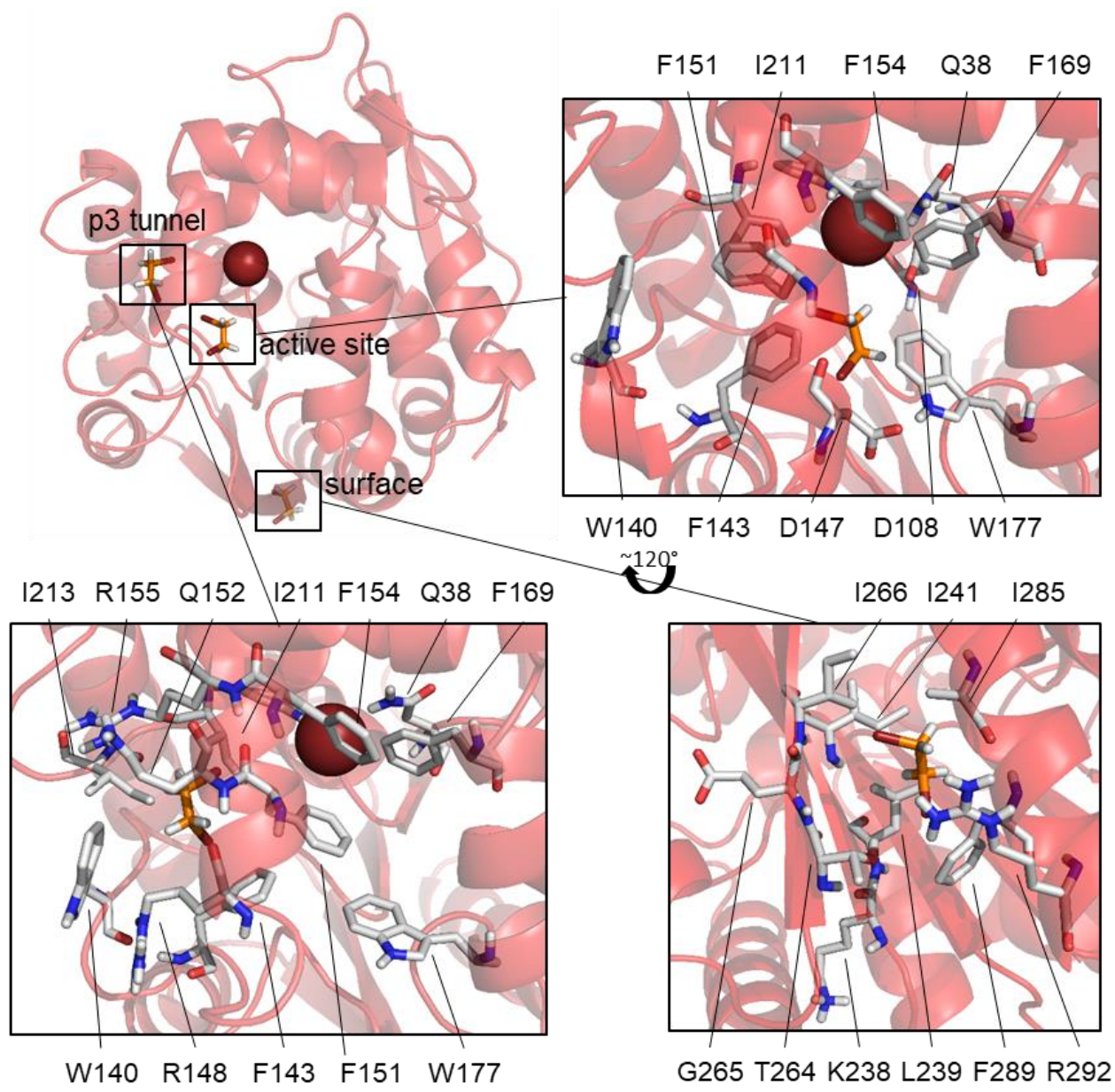

Figure 3. The binding sites for DBE in states that could be responsible for the observed substrate inhibition. Binding at the $p 3$ tunnel would force F151 towards the catalytic site. Binding to the catalytic site would sterically block the exit of Br-. Binding to the surface site could cause substrate inhibition by an allosteric white.

sampling. Around 250000 snapshots were obtained for each system and used to create MSMs with eight Markov states using the binary contact map for the protein's $\mathrm{C} \alpha$ atoms and the atoms of the ligands as the metric (Table 1). The implied timescale plots, Chapman-Kolmogorov test results, and figures depicting the states are presented in the Supplementary Information (Section II).

The starting state (BRE $+\mathrm{Br}^{-}$bound) was identified in all systems other than the WT. This is consistent with the experimental finding that the rate-limiting step of the WT-catalyzed reaction is the release of BRE rather than $\mathrm{Br}^{-}$release. ${ }^{35}$ The WT enzyme also had the greatest number of BRE bound states, further supporting this conclusion.

States with all of the ligands bound or unbound were found for all systems. It is rare $(<3 \%)$ for any system to have all three ligands bound, and it is relatively common for all systems to have all ligands unbound, perhaps except for L177W (6.1\%). With the same exception, the systems were frequently observed in states that could be considered "ready for the next catalytic cycle", i.e. active states in which the only DBE was bound. This state was not observed at all for L177W. The failure to observe the substrate-bound state could be due to the relatively short simulation time or the fact that $\mathrm{L} 177 \mathrm{~W}$ is one of the least catalytically proficient enzymes studied here.

L177W was also the only studied enzyme variant for which states having both $\mathrm{DBE}$ and $\mathrm{Br}^{-}$bound simultaneously were observed, supporting our hypothesized SI mechanism. This variant has a common state with $\mathrm{Br}^{-}$bound and $\mathrm{DBE}$ bound to the surface (31\%), as well as two states where DBE is bound inside the active site (14\%) or the p3 tunnel (17\%) (Figure 3).

The high frequency of the state with DBE bound on the surface and $\mathrm{Br}^{-}$also bound (31\%) suggests that DBE could cause the observed SI via an allosteric mechanism, but there are no modifications to this part of the enzyme that would explain the diminished SI in W140A/F143L/L177W/I211L. Additionally, binding at the same surface site is observed as an uncommon state of W140A/F143L/L177W/I211L (6.8\%) and the WT (BRE bound + DBE on the surface, $11 \%$ ) even though $\mathrm{Br}^{-}$is not bound in these cases. These results suggest that DBE sometimes binds to this part of the enzyme surface but that such binding does not cause $\mathrm{SI}$ because it is also observed in the WT and W140A/F143L/L177W/I211L, both of which exhibit only weak SI. Mutagenesis studies targeting this surface binding site could clarify its effects on the catalytic properties of LinB variants. 
Table 1. The equilibrium probabilities of the Markov states of interest in each system. Probabilities for cases having multiple states with similar descriptions but different ligand orientations are summed and explained in the footnotes. The reader is referred to the supplementary information (section II) for a detailed list of the states and the figures of the Markov states. Probabilities are reported in \%.

\begin{tabular}{|c|c|c|c|c|c|c|}
\hline State & WT & L177W & W140A/L177W & F143L/L177W & L177W/I211L & $\begin{array}{l}\text { W140A/F143/ } \\
\text { L177W/I211L }\end{array}$ \\
\hline All unbound & 20 & 6.1 & 17 & 13 & 37 & 24 \\
\hline All bound & 0.2 & 2.2 & 0.2 & 0.02 & 0.04 & 0.4 \\
\hline \multicolumn{7}{|l|}{ Active state } \\
\hline DBE bound & 30 & & 22 & 50 & 48 & 22 \\
\hline \multicolumn{7}{|l|}{ Possible SI states } \\
\hline $\mathrm{DBE}$ on surface $+\mathrm{Br}$ bound & & 31 & & & & \\
\hline $\mathrm{DBE}+\mathrm{Br}$ bound & & $31^{1}$ & 10 & 0.1 & 0.6 & \\
\hline \multicolumn{7}{|l|}{ Other states } \\
\hline $\begin{array}{l}\mathrm{BRE}+\mathrm{Br} \text { bound + DBE on } \\
\text { surface }\end{array}$ & & 3.9 & 1.2 & & & 6.8 \\
\hline DBE on surface & & 1.8 & & & & \\
\hline $\mathrm{BRE}+\mathrm{Br}$ bound & & & & 16 & 0.2 & \\
\hline $\mathrm{DBE}+\mathrm{BRE}$ bound & $35^{2}$ & & 27 & 15 & 6 & $46^{3}$ \\
\hline BRE bound + DBE on surface & 11 & & & & 5.6 & \\
\hline BRE bound & 4.1 & & 22 & 5.1 & & \\
\hline $\mathrm{Br}$ bound & & 24 & 0.4 & 0.7 & 2.6 & \\
\hline
\end{tabular}

${ }^{1}$ Consists of two states with different binding orientations having probabilities of $17 \%$ and $14 \%$

${ }^{2}$ Consists of two states with different binding orientations having probabilities of $28 \%$ and $5 \%$.

${ }^{3}$ Consists of three states with different binding orientations having probabilities of $39 \%, 5 \%$ and $2 \%$.

The mutations differentiating L177W from W140A/F143L/L177W/I211L are clustered around the p3 tunnel region, which, interestingly, is a binding site for $D B E$ in one L177W state (DBE $+\mathrm{Br}^{-}$bound, $17 \%$, Figure 3 ). This region of L177W should be identical to that in the WT, which does not exhibit strong SI. By looking at the individual Markov states (Supplementary Table S4 and Figure S4-9), we can see that both DBE and BRE can bind to this binding site in the WT, but such binding is rare $(<6 \%)$. The $\mathrm{Br}^{-}$bound states may occur frequently in L177W because the L177W mutation hinders the exit of reaction products; the binding of DBE in this site could further hinder the exit of $\mathrm{Br}^{-}$. Figure 3 shows that the binding of DBE to the $\mathrm{p} 3$ tunnel forces F151 to face the active site, creating yet another a steric barrier to the exit of $\mathrm{Br}$. In the third possible SI state, DBE binds to the active site (Figure 3 ) and interacts with the mutated L177W. Since DBE occupies the active site, it sterically blocks the exit of $\mathrm{Br}^{-}$and affects the interactions of water molecules involved in releasing $\mathrm{Br}^{-}$from the halidestabilizing residues.

We next examined possible SI states in variants that combine L177W with individual mutations from the W140A/F143L/L177W/I211L quadruple mutant. In F143L/L177W and L177W/I211L, the states responsible for SI (DBE bound at the active site or p3 tunnel) are very rarely observed (<1\% probability). W140A/L177W, on the other hand, often adopts a state with $\mathrm{DBE}$ and $\mathrm{Br}$ - bound (10\%). In this state, DBE interacts with both binding sites observed for L177W, i.e. the active site and the $\mathrm{p} 3$ tunnel region (Figure $\mathbf{3}$ ), suggesting a possible enhancement of the SI effect. Additionally, the most common states of $\mathrm{F} 143 \mathrm{~L} / \mathrm{L} 177 \mathrm{~W}$ and L177W/I211L resemble those of the catalytically efficient WT enzyme and W140A/F143L/L177W/I211L, and are thus expected to have greater catalytic activity than W140A/L177W.

Based on the MSM results, the following predictions can be made for L177W/I211L, W140A/L177W and F143L/L177W: (i) L177W/I211L and F143L/L177W should display weaker SI than L177W, (ii) W140A/L177W should display the strongest SI of the three double-point variants, and (iii) F143L/L177W and L177W/I211L should be more effective than W140A/L177W in the transport of ligands and thus also in catalysis.

\section{Mutagenesis of $p 3$ tunnel residues}

To test the MSM-based predictions against experiment and to dissect the importance of individual p3 tunnel mutations in W140A/F143L/L177W/I211L, we constructed and characterized three additional LinB double-point variants: W140A/L177W, F143L/L177W, and L177W/I211L (Supplementary Information Section III). The equilibrium dissociation constants $\left(K_{\mathrm{SI}}\right)$ estimated for these variants using steady-state kinetic data showed (Table 2) that compared to L177W, the substrate affinity of the inhibitory complex precursor was reduced in F143L/L177W and L177W/I211L but significantly enhanced in W140A/L177W. These findings agree well with the simulations, 
Table 2. Steady-state kinetic parameters for LinB variants with engineered access tunnels. All steady-state experiments were performed at $37^{\circ} \mathrm{C}$ and $\mathrm{pH} 8.6$.

\begin{tabular}{|c|c|c|c|c|c|}
\hline Variant & $K_{\mathrm{m}}(\mathrm{mM})$ & $k_{\text {cat }}\left(\mathbf{s}^{-1}\right)$ & $K_{\mathrm{sI}}(\mathrm{mM})$ & $k_{\text {cat }} / K_{\mathrm{m}}\left(\mathrm{mM}^{-1} \mathrm{~s}^{-1}\right)$ & $K_{\mathrm{sl}} / K_{\mathrm{m}}$ \\
\hline WT $^{1}$ & $1.7 \pm 0.2$ & $12 \pm 4$ & $12.4 \pm 0.3$ & $7 \pm 2$ & $7.3 \pm 0.9$ \\
\hline $\mathrm{L}^{177 W^{1}}$ & $0.42 \pm 0.1$ & $3.1 \pm 0.1$ & $1.34 \pm 0.03$ & $7 \pm 2$ & $3.2 \pm 0.8$ \\
\hline W140A/F143L/L177W/I211L ${ }^{1}$ & $2.35 \pm 0.03$ & $57 \pm 3$ & $7.3 \pm 0.1$ & $24 \pm 1$ & $3.1 \pm 0.1$ \\
\hline W140A/L177W & $4.5 \pm 0.9$ & $7 \pm 1$ & $0.39 \pm 0.06$ & $1.6 \pm 0.4$ & $0.09 \pm 0.02$ \\
\hline F143L/L177W & $2.1 \pm 0.1$ & $16 \pm 1$ & $2.06 \pm 0.01$ & $7.6 \pm 0.6$ & $0.98 \pm 0.05$ \\
\hline L177W/I211L & $0.12 \pm 0.01$ & $2.33 \pm 0.04$ & $1.9 \pm 0.4$ & $19 \pm 2$ & $16 \pm 4$ \\
\hline I211L & $0.76 \pm 0.04$ & $8.3 \pm 0,2$ & $4.6 \pm 0.1$ & $11 \pm 1$ & $6.1 \pm 0.3$ \\
\hline F143L & $1.56 \pm 0.05$ & $2.1 \pm 0.1$ & $1.8 \pm 0.2$ & $1.3 \pm 0.1$ & $1.2 \pm 0.1$ \\
\hline W140A/F143L/1211L ${ }^{2}$ & $3.9 \pm 1.7$ & $3.8 \pm 0.1$ & $23 \pm 3$ & $1.0 \pm 0.4$ & $6 \pm 3$ \\
\hline
\end{tabular}

${ }^{1}$ Steady-state kinetic constants adopted from Brezovsky et al. 2016. ${ }^{36}$

2 Steady-state kinetic constants adopted from Kokkonen et al. 2019. ${ }^{35}$

which predicted that W140A/L177W would show the strongest SI effect among the tested double-point variants.

The kinetic data also agree well with the computational predictions of the catalytic efficiency: F143L/L177W and $\mathrm{L} 177 \mathrm{~W} / \mathrm{I} 211 \mathrm{~L}$ were expected to frequently exist in the active state (the probabilities of DBE bound-states were $50 \%$ and $48 \%$ for $\mathrm{F} 143 \mathrm{~L} / \mathrm{L} 177 \mathrm{~W}$ and $\mathrm{L} 177 \mathrm{~W} / \mathrm{I211L}$, respectively) and accordingly had a significantly higher specificity constant $k_{\text {cat }} / K_{\mathrm{m}}$ than W140A/L177W, for which the frequency of the active DBEbound state was rather low (22\%). The overall impact of substrate inhibition depends on the relative magnitude of the Michaelis constant $K_{\mathrm{m}}$ and $K_{\mathrm{SI}}$. The estimated $K_{\mathrm{SI}} / K_{\mathrm{m}}$ ratios (Table 2) are consistent with strong SI in W140A/L177W $\left(K_{\mathrm{sl}} / K_{\mathrm{m}}\right.$ $=0.09 \pm 0.02)$ and also indicate significant attenuation of inhibition in L177W/I211L $\left(K_{\mathrm{sl}} / K_{\mathrm{m}}=16 \pm 4\right)$, even compared to the WT $\left(K_{\mathrm{s}} / K_{\mathrm{m}}=7.3 \pm 0.9\right)$. Moreover, L177W/1211L had the highest specificity constant of any tested variant $\left(k_{\text {cat }} / K_{\mathrm{m}}=19 \pm\right.$ $\left.2 \mathrm{mM}^{-1} \mathrm{~s}^{-1}\right)$, suggesting that the $1211 \mathrm{~L}$ substitution contributes greatly to the unusual efficiency of W140A/F143L/L177W/I211L $\left(k_{\text {cat }} / K_{\mathrm{m}}=24 \pm 2 \mathrm{mM}^{-1} \mathrm{~s}^{-1}\right)$.

We also constructed the single-point p3 tunnel variants W140A, F143L and 1211L to test their possible synergistic effects with L177W. Only two of these variants, F143L and 1211L, were successfully expressed and purified for kinetic analysis. Relative to the WT, F143L exhibited reduced catalytic efficiency and weaker SI. $1211 \mathrm{~L}$ showed a similar kinetic impact of SI $\left(K_{\mathrm{SI}} / K_{\mathrm{m}}=\right.$ $6.1 \pm 0.3)$ and partially improved the catalytic efficiency $\left(k_{\text {cat }} / K_{\mathrm{m}}\right.$ $=11 \pm 1 \mathrm{mM}^{-1} \mathrm{~s}^{-1}$ ), but none of these parameters reached the level obtained for $\mathrm{L} 177 \mathrm{~W} / \mathrm{I} 211 \mathrm{~L}$. These results show that the presence of Trp at the position 177 has important effects on the catalytic performance of W140A/F143L/L177W/1211L $\left(k_{\text {cat }} / K_{\mathrm{m}}=\right.$ $\left.24 \pm 1 \mathrm{mM}^{-1} \mathrm{~s}^{-1}\right)$ and L177W/I211L $\left(k_{\mathrm{cat}} / K_{\mathrm{m}}=19 \pm 2 \mathrm{mM}^{-1} \mathrm{~s}^{-1}\right)$. A similar effect was observed for the back mutation at position 177 leading to W140A/F143L/I211L with $k_{\text {cat }} / K_{m}=1.0 \pm 0.4 \mathrm{mM}^{-}$ $1 \mathrm{~s}^{-1}$. Moreover, the systematic mutagenesis showed an interesting synergism between L177W and 1211L, which significantly suppressed the SI $\left(K_{\mathrm{SI}} / K_{\mathrm{m}}=16 \pm 4\right)$, although these mutations had a rather opposite effect when introduced separately $\left(K_{\mathrm{sl}} / K_{\mathrm{m}}=3.2 \pm 0.8\right.$ and $6.1 \pm 0.3$ for $\mathrm{L} 177 \mathrm{~W}$ and $1211 \mathrm{~L}$, respectively).

\section{Multivariate data analysis}

A partial least squares (PLS) analysis was performed to test how well the theoretical assumptions and models of the molecular dynamics simulations reproduced the macroscopic experimental data (Supplementary Information Section IV). The PLS analysis yielded a statistically significant model correlating the probabilities of the Markov states calculated based on the molecular dynamics simulations and the logarithm of the experimentally determined ratio of the inhibition constant and the Michaelis-Menten constant $\left(\log K_{\mathrm{i}} / K_{\mathrm{m}}\right)$. A model based on two principal components had a significant coefficient of determination $\left(R^{2}=0.84\right)$ and a cross-validation coefficient of $Q^{2}=0.68$. The small difference between $R^{2}$ and $Q^{2}$ confirms that the model is not over-fitted. Significant variables contributing to the explanation of the experimental $K_{\mathrm{i}} / K_{\mathrm{m}}$ values were BRE_bound, BRE_bound+DBE_surface, BR_bound and All_unbound. A permutation test using 300 rounds of permutation of the $y$-components significantly reduced both $R^{2}$ and $\mathrm{Q}^{2}$ because the correlation between the permutated and original data weakened, implying that the obtained values are not due to pure chance (Supplementary Figure S13). Because of the small number of observations, Monte Carlo simulations were performed to test the validity of the permutation analysis. Ten thousand data sets of the same size as in our analysis were generated by drawing randomly and independently from a uniform distribution. These data sets were then subjected to the same PLS analysis protocol and their $R^{2}$ and $Q^{2}$ values were computed. In addition, the PLS was repeated for each dataset using only the 3 variables with the best VIP values of the 12 available. While there were several instances in which the $R^{2}$ coefficient for random datasets exceeded that for the real data, the small $p$-values for the coefficient $Q^{2}$ (Supplementary Figure S14) indicate that the observed $Q^{2}$ of 0.68 would be unlikely to be observed by chance even given the small sample size. We thus conclude that our theoretical models based on the 
molecular dynamic simulations reproduced the experimental kinetic observations well.

\section{DISCUSSION}

Substituting the L177 position of LinB with a bulky residue (L177W) introduced a strong SI effect into the enzyme's catalytic cycle. This mutation reduces the size of the access tunnel and catalytic site, making it implausible that the SI was due to the formation of an unproductive complex resulting from an increase in the number of substrate molecules bound simultaneously at the catalytic site. Thus, as expected, the kinetic simulations produced unrealistic values when trying to fit the data to comply with this classical theory. No allosteric binding sites were found on LinB that might explain the observed SI, although we did observe a possible substratebinding site at the surface (Figure 3). However, non-specific binding of DBE to this region was observed in almost all studied LinB variants.

The global kinetic analysis was used to systematically test three possible models of SI. In addition to Haldane's traditional mechanism including an enzyme complex with two substrate molecules, the binding of the substrate to an alkyl-enzyme intermediate and the enzyme-product complex were evaluated kinetically. The simultaneous analysis of steady-state and transient kinetic data using numerical integration made it possible to distinguish between the expected models and suggested that the observed $\mathrm{SI}$ is due to the formation of an unproductive enzyme-product-substrate complex. In particular, the transient-state burst analysis strongly supported this inhibition mechanism. Clear discrimination between the different inhibition models was achieved upon comparing the experimentally observed and numerically simulated amplitudes for the bursts of the halide and alcohol products, which corresponded to the fractions of the alkyl-enzyme intermediate and enzyme-product-complex available during the catalytic cycle. The only model consistent with the rates and amplitudes observed in all of the kinetic experiments was that in which the substrate binds to the enzyme-product complex, leading to an unproductive form. Since halide release is a slow rate-limiting step, ${ }^{36,57}$ the enzyme-bromide complex accumulates strongly during the conversion of DBE catalyzed by L177W. This makes the enzyme-product complex the main target for potential inhibition.

Mechanistically, SI in L177W may occur because DBE binds to the catalytic site and presents a steric barrier to the exit of $\mathrm{Br}^{-}$. The binding of DBE to the p3 tunnel could not induce SI via direct steric blockage because in this case, DBE would only block a rarely-used auxiliary tunnel. ${ }^{36}$ However, the binding of DBE to this position forces F151 to face the catalytic site, creating a steric blockage similar to that formed when DBE binds to the catalytic site. The $\mathrm{W} 177$ residue in the L177W variant forms a parallel displaced $\pi$-stacking interaction with $\mathrm{F} 151$, stabilizing the blocking conformation. This conformation of F151 is also stabilized via T-shaped $\pi$ stacking with F143, which may partly explain the loss of SI in the F143L/L177W variant.
The other possible mechanism of SI by DBE is based on locking the W177 residue of L177W into the closed conformation. We have previously shown that L177W undergoes slow conformational changes between open and closed states and that it is difficult for $\mathrm{Br}^{-}$to exit from the closed conformation. ${ }^{35}$ W140A/F143L/L177W/I211L displays similar conformational dynamics but over shorter time scales. When DBE is bound to the p3 tunnel or the active site, it forms favorable lipophilic interactions with the surrounding residues, reducing the likelihood that the protein will go from the closed to the open state.

The mutations at the p3 tunnel (W140A/F143L/I211L) widened the tunnel and reduced the number of possible lipophilic interactions, thereby reducing the site's affinity for DBE. This can be seen in the Markov states of L177W-containing doublepoint variants: only $\mathrm{W} 140 \mathrm{~A} / \mathrm{L} 177 \mathrm{~W}$ has a relatively common SI state like L177W. The experimental results confirmed that the double-point mutants generally had weaker SI than L177W. Additionally, the experimental data showed that the W140A mutation causes structural instability, possibly because too many hydrophobic contacts are lost in the region responsible for the conformational dynamics of L177W and W140A/F143L/L177W/I211L (for a more detailed discussion, see our previous manuscript ${ }^{35}$ ). Additionally, systematic mutagenesis of $\mathrm{p} 3$ tunnel residues revealed an unusual synergy between L177W and 1211L, which are key residues providing high catalytic efficiency and reduced substrate inhibition. The essential role of $\mathrm{L} 177 \mathrm{~W}$ was previously tested by back-mutation of $\mathrm{W} 140 \mathrm{~A} / \mathrm{F} 143 \mathrm{~L} / \mathrm{L} 177 \mathrm{~W} / \mathrm{I} 211 \mathrm{~L}$ in the position $177 .{ }^{36}$ The W140A/F143L/I211L variant completely lacked the high catalytic efficiency of W140A/F143L/L177W/I211L. Similarly, the efficiency of L177W/I211L was not replicated by either the L177W or the $1211 \mathrm{~L}$ substitutions individually. Interestingly, the synergistic substitutions $\mathrm{L} 177 \mathrm{~W}$ and $1211 \mathrm{~L}$ are located in two different access tunnels in remote parts of the protein.

\section{CONCLUSIONS}

We have shown that SI can be caused by a substrate molecule blocking the exit of the product from the enzyme-product complex. This mechanism is not typically considered for single substrate reactions. Additionally, we have shown that single amino acid substitutions in enzyme access tunnels can introduce or weaken SI. This effect should therefore be taken into account during enzyme engineering studies. Experimental analysis including studies on transient kinetics can identify the specific stages of the catalytic cycle affected by SI. This information can be then used to conduct molecular dynamics simulations with MSMs to clarify the SI mechanism at the molecular level. Once a mechanism is established, rational protein engineering targeting the relevant states can be undertaken to reduce the probability of undesirable SI. This strategy should be generally applicable to many other enzyme systems with available structural information. 


\section{ACKNOWLEDGEMENTS}

The authors would like to thank the RECETOX research infrastructure (the Czech Ministry of Education, Youth and Sports: LM2018121) and Teaming project: CETOCOEN EXCELLENCE Teaming 2 project supported by Horizon2020 (857560) and the Czech Ministry of Education, Youth and Sports (02.1.01/0.0/0.0/18_046/0015975). Computational resources were supplied by the project "e-Infrastruktura CZ" (e-INFRA LM2018140) provided within the program Projects of Large Research, Development and Innovations Infrastructures. PK and $A B$ are supported by the Operational Programme Research, Development and Education - Projects „MSCAfellow@MUNI“ CZ.02.2.69/0.0/0.0/17_050/0008496 CZ.02.2.69/0.0/0.0/19_074/0012727, respectively.

\section{CONFLICTS OF INTEREST}

There are no conflicts of interest to declare.

\section{REFERENCES}

1 M. C. Reed, A. Lieb and H. F. Nijhout, BioEssays, 2010, 32, $422-$ 429.

2 B. Wu, Drug Metab. Rev., 2011, 43, 440-456.

3 M. Yoshino and K. Murakami, Springerplus, 2015, 4, 292.

4 A. W. Fenton and G. D. Reinhart, Biochemistry, 2003, 42, 12676 12681

5 J. B. S. Haldane, Reprint by MIT, Cambridge, 1965, 84.

6 Y. Lin, P. Lu, C. Tang, Q. Mei, G. Sandig, A. D. Rodrigues, T. H. Rushmore and M. Shou, Drug Metab. Dispos., 2001, 29, 368-374.

7 C. Chen, J. C. Joo, G. Brown, E. Stolnikova, A. S. Halavaty, A Savchenko, W. F. Anderson and A. F. Yakunin, Appl Environ Microbiol, 2014, 80, 3992-4002.

8 K. D. Tidemand, G. H. Peters, P. Harris, E. Stensgaard and H. E. M. Christensen, Biochemistry, 2017, 56, 6155-6164.

9 J. R. Warner, L. S. Behlen and S. D. Copley, Biochemistry, 2008, 47, 3258-3265.

10 E. H. Jang, S. A. Park, Y. M. Chi and K. S. Lee, Biochemical and Biophysical Research Communications, 2015, 461, 487-493.

11 G. W. Heberlig, M. Wirz, M. Wang and C. N. Boddy, Org. Lett., 2014, 16, 5858-5861.

12 P. Kallio, P. Patrikainen, G. A. Belogurov, P. Mäntsälä, K. Yang, J. Niemi and M. Metsä-Ketelä, Biochemistry, 2013, 52, 45074516.

13 A. E. Leroux, J. R. Haanstra, B. M. Bakker and R. L. KrauthSiegel, J Biol Chem, 2013, 288, 23751-23764.

14 T. Liu, Q. Wu, L. Liu and Q. Yang, Process Biochemistry, 2013 , 48, 103-108.

15 A. Tramonti, C. Nardella, M. L. di Salvo, A. Barile, F. Cutruzzolà and R. Contestabile, Biochemistry, 2018, 57, 6984-6996.

16 P. Mehrabi, C. Di Pietrantonio, T. H. Kim, A. Sljoka, K. Taverner, C. Ing, N. Kruglyak, R. Pomès, E. F. Pai and R. S. Prosser, J. Am. Chem. Soc., 2019, 141, 11540-11556.

17 I. L. Mainprize, J. D. Bean, C. Bouwman, M. S. Kimber and C. Whitfield, J. Biol. Chem., 2013, 288, 23064-23074.

18 R. A. G. Reis, F. Salvi, I. Williams and G. Gadda, Biochemistry, 2019, 58, 2594-2607.

19 F. B. H. Rehm, M. A. Jackson, E. D. Geyter, K. Yap, E. K Gilding, T. Durek and D. J. Craik, PNAS, 2019, 116, 7831-7836.

20 I. N. Kiburu and N. LaRonde-LeBlanc, PLOS ONE, 2012, 7, e37371.

21 L. Jin, K. Yang, K. Yao, S. Zhang, H. Tao, S.-T. Lee, Z. Liu and R. Peng, ACS Nano, 2012, 6, 4864-4875.
22 H. Jahić, C. F. Liu, J. Thresher, S. Livchak, H. Wang and D. E. Ehmann, Biochemical Pharmacology, 2012, 84, 654-660.

23 W. Fang, T. Du, O. G. Raimi, R. Hurtado-Guerrero, M. D. Urbaniak, A. F. M. Ibrahim, M. A. J. Ferguson, C. Jin and D. M. F. van Aalten, Molecular Microbiology, 2013, 89, 479-493.

24 J. Wang, J. Zhu, C. Min and S. Wu, BMC Biotechnology, 2014, 14, 40 .

25 Y.-L. Tao, D.-H. Yang, Y.-T. Zhang, Y. Zhang, Z.-Q. Wang, Y.S. Wang, S.-Q. Cai and S.-L. Liu, Appl Microbiol Biotechnol, 2014, 98, 2519-2531.

26 J. J. Sánchez-Romero and L. F. Olguin, Biochemistry and Biophysics Reports, 2015, 3, 161-168.

27 S. Reich, D. Guilligay and S. Cusack, Nucleic Acids Res, 2017, 45, 3353-3368.

28 K. A. Johnson, in Wiley Encyclopedia of Chemical Biology, American Cancer Society, 2008, pp. 1-8.

29 K. A. Johnson, in The Enzymes, ed. D. S. Sigman, Academic Press, 1992, vol. 20, pp. 1-61.

$30 \mathrm{H}$. Hu, C. Luo and Y. G. Zheng, J Biol Chem, 2016, 291, 26722 26738.

31 M. T. Kamlay and J. D. Shore, Arch. Biochem. Biophys., 1983, 222, 59-66.

32 Y. Wei, P. Kuzmič, R. Yu, G. Modi and L. Hedstrom, Biochemistry, 2016, 55, 5279-5288.

33 H. J. Fromm and D. R. Nelson, J. Biol. Chem., 1962, 237, 215220.

34 D. B. Northrop and W. W. Cleland, J. Biol. Chem., 1974, 249, 2928-2931.

35 P. Kokkonen, J. Sykora, Z. Prokop, A. Ghose, D. Bednar, M. Amaro, K. Beerens, S. Bidmanova, M. Slanska, J. Brezovsky, J. Damborsky and M. Hof, J. Am. Chem. Soc., 2018, 140, 1799918008.

36 J. Brezovsky, P. Babkova, O. Degtjarik, A. Fortova, A. Gora, I. Iermak, P. Rezacova, P. Dvorak, I. K. Smatanova, Z. Prokop, R. Chaloupkova and J. Damborsky, ACS Catal., 2016, 6, 7597-7610.

37 P. W. Rose, C. Bi, W. F. Bluhm, C. H. Christie, D. Dimitropoulos, S. Dutta, R. K. Green, D. S. Goodsell, A. Prlic, M. Quesada, G. B. Quinn, A. G. Ramos, J. D. Westbrook, J. Young, C. Zardecki, H. M. Berman and P. E. Bourne, Nucleic Acids Research, 2013, 41, D475-D482.

38 J. J. Gehret, L. Gu, T. W. Geders, W. C. Brown, L. Gerwick, W. H. Gerwick, D. H. Sherman and J. L. Smith, Protein Sci., 2012, 21, 239-248

39 A. J. Oakley, M. Klvana, M. Otyepka, Y. Nagata, M. C. J. Wilce and J. Damborský, Biochemistry, 2004, 43, 870-878.

40 R. Anandakrishnan, B. Aguilar and A. V. Onufriev, Nucleic Acids Research, 2012, 40, W537-W541.

41 The PyMOL Molecular Graphics System, Version 1.7.4 Schrödinger, LLC.

42 S. Doerr, M. J. Harvey, F. Noé and G. De Fabritiis, Journal of Chemical Theory and Computation, 2016, 12, 1845-1852.

43 I. Leontyev and A. Stuchebrukhov, Phys Chem Chem Phys, 2011, 13, 2613-2626.

44 M. J. Harvey and G. De Fabritiis, J Chem Theory Comput, 2009, 5, 2371-2377.

45 M. J. Harvey, G. Giupponi and G. D. Fabritiis, J. Chem. Theory Comput., 2009, 5, 1632-1639.

46 K. A. Feenstra, B. Hess and H. J. C. Berendsen, J. Comput. Chem., 1999, 20, 786-798.

47 C. W. Hopkins, S. Le Grand, R. C. Walker and A. E. Roitberg, J. Chem. Theory Comput., 2015, 11, 1864-1874.

48 Y. Naritomi and S. Fuchigami, J Chem Phys, 2011, 134, 065101.

49 A. Höskuldsson, Journal of Chemometrics, 1988, 2, 211-228.

50 S. Wold, E. Johansson and M. Cocchi, in 3D QSAR in Drug Design. Theory, Methods, and Applications, ESCOM Science Publisher, Leiden, vol. 1993, pp. 523-550. 
51 S. Wold and W. J. Dunn, J. Chem. Inf. Comput. Sci., 1983, 23, 613.

52 S. Wold, Quantitative Structure-Activity Relationships, 1991, 10, 191-193.

53 I. Iwasaki, S. Utsumi and T. Ozawa, BCSJ, 1952, 25, 226-226.

54 M. J. Todd and J. Gomez, Anal. Biochem., 2001, 296, 179-187.

55 K. A. Johnson, Z. B. Simpson and T. Blom, Anal. Biochem., 2009, 387, 20-29.

56 K. H .G. Verschueren, F. Seljée, H. J. Rozeboom, K. H. Kalk and B. W. Dijkstra. Nature, 1993, 363, 693-698.

57 L. Biedermannová, Z. Prokop, A. Gora, E. Chovancová, M. Kovács, J. Damborsky and R. C. Wade, J. Biol. Chem., 2012, 287, 29062-29074. 